\title{
A Component-Based Adaptive Model for Context-Awareness in Ubiquitous Computing
}

\author{
Soo-Joong Ghim ${ }^{1}$, Yong-Ik Yoon ${ }^{1}$, and Ilkyeun $\mathrm{Ra}^{2}$ \\ ${ }^{1}$ Department of Computer Science, Sookmyung Women's University, \\ Chungpa-Dong 2-Ga, Yongsan-Gu, 140-742, Seoul, Korea \\ \{yiyoon, sjghim\} @sookmyung.ac.kr \\ ${ }^{2}$ Department of Computer Science \& Engineering, \\ University of Colorado, Denver and Health Sciences Center, \\ Denver, CO 80217, U.S.A. \\ ikra@carbon. cudenver.edu
}

\begin{abstract}
A high adaptable middleware has been an essential platform to provide more flexible services for multimedia, mobile, and ubiquitous applications in ubiquitous computing environments. In addition, the persistent services of these application systems and their middleware in ubiquitous computing are required so that they can be aware of the frequent and unpredictable changes in users' requirements as well as environmental conditions and adapt their behavioural changes. However, current approaches for supporting adaptability have made applications themselves trigger and execute an adaptive mechanism when the underling infrastructure notifies them about any changes. In this paper, we propose a novel component-based contextadaptive model for context-awareness middleware to support efficiently dynamic adaptation of application services. We also demonstrate the current implementation of the context-adaptive middleware that help applications to adapt their ubiquitous computing environments according to rapidly changing contexts such as user-specific preferences, application-specific preferences, and low-level configurations.
\end{abstract}

\section{Introduction}

Ubiquitous computing allows application developers to build a large and complex distributed system that can transform physical spaces into computationally active and intelligent environments [2]. Ubiquitous applications need a middleware that can detect and act upon any context changes created by the result of any interactions between users, applications, and surrounding computing environment for applications without users' interventions [1]. The context-awareness has become the one of core technologies for application services in ubiquitous computing environment and been considered as the indispensable function for ubiquitous computing applications. In order to provide context-awareness services, underlying platforms in ubiquitous computing should be able to recognize contextual changes so that applications use contexts for evaluating new environments and finding an appropriate action by the result of evaluation for these changes. 
Previous context-awareness researches have been mainly focusing on the location change of users or devices in context-awareness related fields [1,6]. In ubiquitous computing environment, however, devices or software services can be added to or removed from the system at anytime, and also contexts or preferences of users are changing frequently. Thus, the mobile applications are expected to be capable of adapting their behaviors to ensure they can continue to offer the best possible level of service to their users. The required level of a system's adaptation can be ranged from the operating system up to the application level.

Supporting high adaptability is an essential requirement for ubiquitous computing systems because the ubiquitous computing environments is highly dynamic, and characterized by frequent and unpredictable changes in different contexts. More generally, the adaptation can be applied to a wide range of aspects at different levels of the system. These include the communications aspects as well as several issues such as allocating resources to the set of activities and external services currently being used. Thus, this adaptation should be driven by awareness of a wide range of issues including its communication performance, resource usage, location, cost, and preference [3]. The extreme emphasis on adaptation at specific level can cause some problems, for example, the adaptation at the operating system level can serious affect on its integrity and performance. In opposite case, leaving all adaptations to the application level would impose a heavy burden on application programmers.

The current approach for supporting adaptable services or applications is based on the classic layered architectural model where adaptation is provided at the various layers (data link, network, transport or application layers) in isolation [4]. Due to the very limited information being shared across protocol layers, adaptation strategies are uni-dimensional - i.e. they only consider one parameter at a time - and often are leading to unsatisfactory results [5]. Another limitation of current approaches is that applications themselves are responsible for triggering and executing adaptive mechanism when the underling infrastructure notifies them about any changes [6].

In this paper, we present a novel adaptive model for context-awareness middleware framework that can overcome the current limitations and support persistence services that allow mobile applications to operate well balanced adaptations level between system and application, and customize the adaptation services for contextual changes. We also describe a component-based context-adaptive model to manage dynamic adaptation in contextual changes. The rest of this paper is organized as follows: Section 2 proposes a component-based framework to support adaptability for mobile agents. In section 3 we explain our conceptual context-adaptive model and component model for ubiquitous computing. Section 4 shows how adaptations are triggered by a policy and explain the detail implementations for audio service for PDA users. Section 5 concludes our research results and discusses future works.

\section{A Context-Adaptive Model}

\subsection{Definition and Classification of Context}

A term called context has different meaning, and is used extensively. Meaning of a context has been used in many various research fields such as operating systems, programming language, artificial intelligences, and natural language processing. 
Moreover, the meaning of context in a user interface design is very different from those areas. It, however, has been agreed on that a context is the key concept for mobile application systems in ubiquitous computing, although it is differently understood from and used by other various fields.

In this paper, we define contexts as environmental information that may cause to activate adaptation mechanisms and as requirements of users or applications. We classify contexts into two levels: high-level and low-level context in Figure 1. The high-level contexts include the preference of users and applications. The preference is the explicit requirements about resources and quality of services. The resources preference is a use degree of resources that are requested by users or applications. The quality of service preference includes display elements such as resolution, a frame rate, and users' security requirements. The low-level contexts are subdivided into the user configuration and resources configuration. The user configuration consists of users' device, location, time. The resource configuration is systems' memory size, computing power, and network bandwidth.

Current existing other research efforts have considered the user preference as a kind of the information that a user would like to get information or contents, and can be used to supply appropriate information to the user through filtering by similarity measurement. However, we take into consideration of characteristics of ubiquitous computing, and we define the user preference as 'the explicit requirements for contexts' in order to adapt dynamically delivery of services when context changes are occurred.

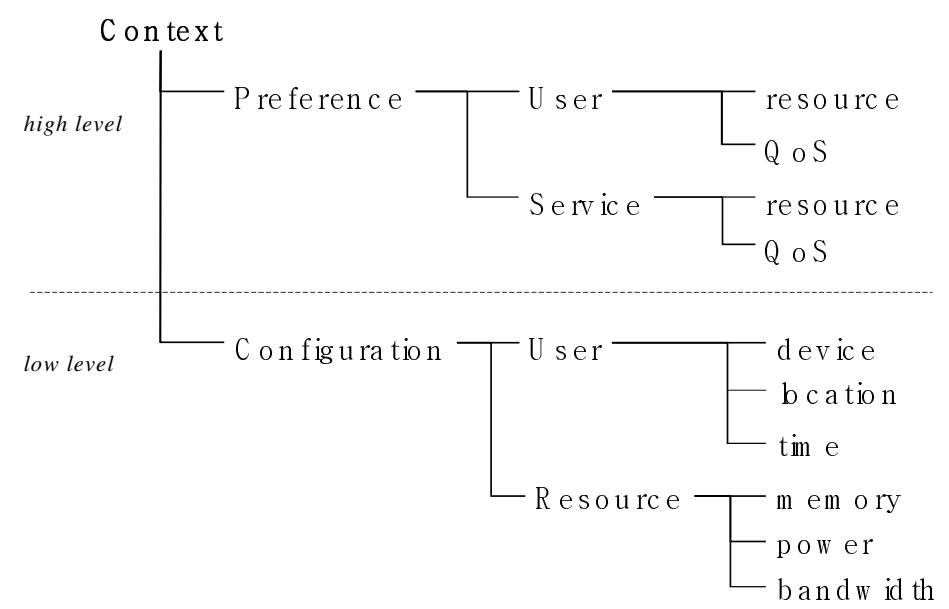

Fig. 1. Classification of context

\subsection{Conceptual Model}

\subsubsection{Adaptation}

Our context-adaptive model defines several elements for supporting contextawareness and adaptation methods. The detail relationships between elements of the context-adaptive model are shown in Figure 2. 


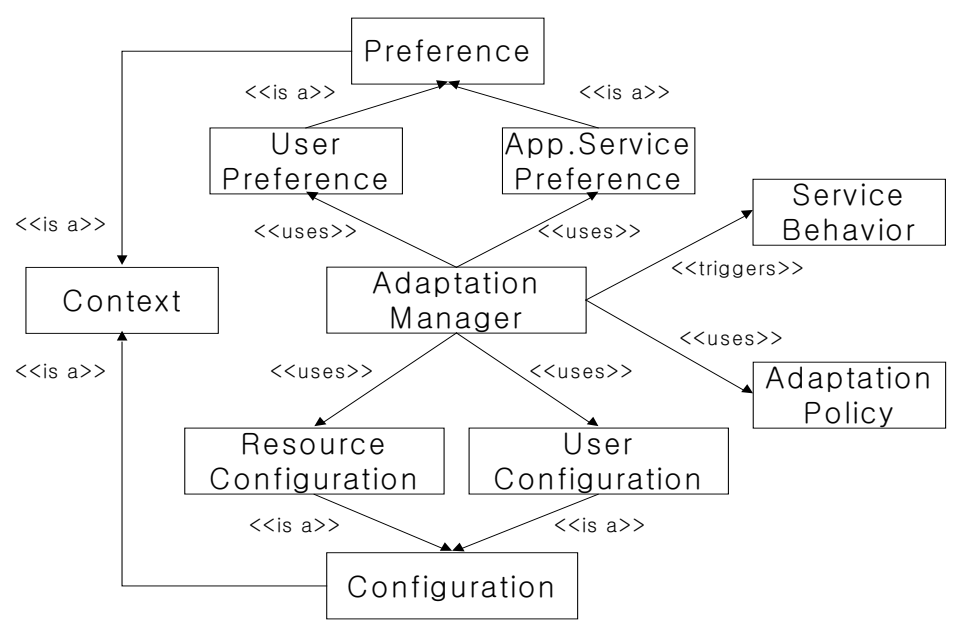

Fig. 2. Adaptation model

As shown in Figure 2, both the user preference and the application preference are considered as the explicit requirements for the usage of resources. We number the associative relationship between these preferences in order to decide on how to provide application services that can maximize satisfaction of user requirements. The configuration information includes a user's device types, locations, time, resources, context of user devices, and adaptation policies that specify association rules between contextual changes of resources and application service behaviours. In our model, the adaptation method reflects preference and configurations, and satisfies user requirements that have user environments to be safe and operational.

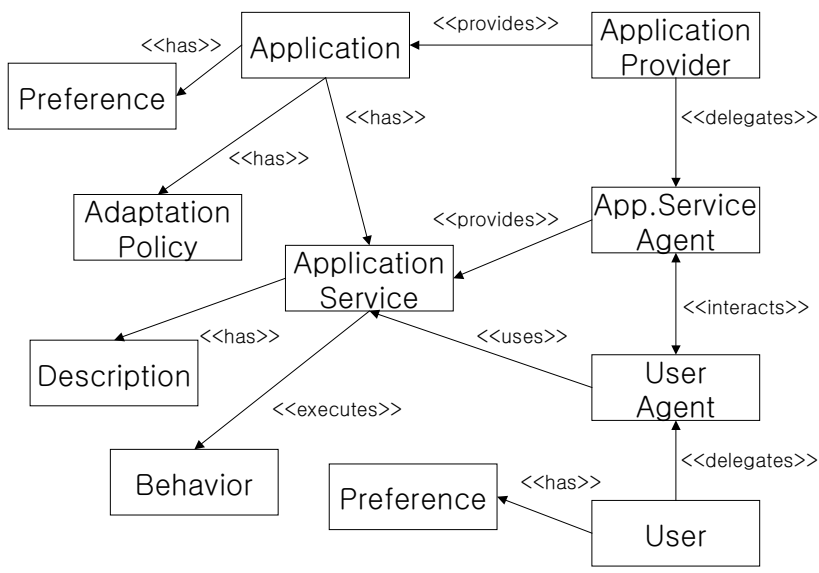

Fig. 3. Application service model 


\subsubsection{Application Service}

Our application service model emphasizes on individual behaviours that compose application services, and establish details related to application service offering as shown in Figure 3.

An application service is the abstract concept of a task to be performed, and can be expressed with the description about a name and functions. The functionality of task is realized by service behaviours that can be composed of operations or methods that are invoked by an agent. In this model, an application consists of application services, adaptation polices and preference for users and services. The adaptation polices in an application are used for the context adaptation.

\section{Dynamic Context-Adaptation}

\subsection{Adaptation Policy}

The adaptation policy is a set of rules for changing application service behaviors with respect to different contexts, and is a key feature for the triggered adaptation. The triggered adaptation does not depend on only the notification mechanism from an underlying system as a voluntary adaptation way of an application about a context. It depends upon the self and dynamic detection for changes in an application behavior according to contextual changes or an application's needs related to controlling an adaptation process.

To support context-awareness, it is required to process low-level tasks including periodic monitoring and detection of changes. A middleware can conceal the complexity with carrying out this task, and can decrease a burden of application developer. However, applications should inform a middleware about which a service should be adapted, and which a behavior should be triggered for each specific context when applications are being instantiated because it is difficult for a middleware to predict various changes of each context in requirements of an application.

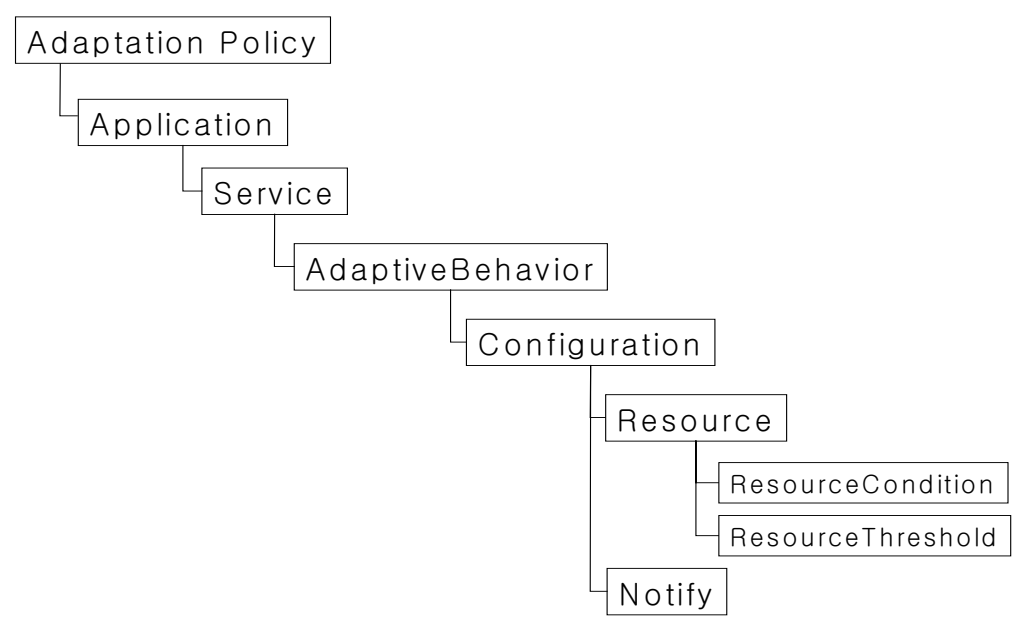

Fig. 4. Adaptation policy hierarchy 
As shown in Figure 4, the adaptation policy describes important information for application services, required configuration in offering services, and behaviors to be triggered when certain context changes occur. A middleware would decide which rule is to apply for service delivery in current context using adaptation policy when application service is requested.

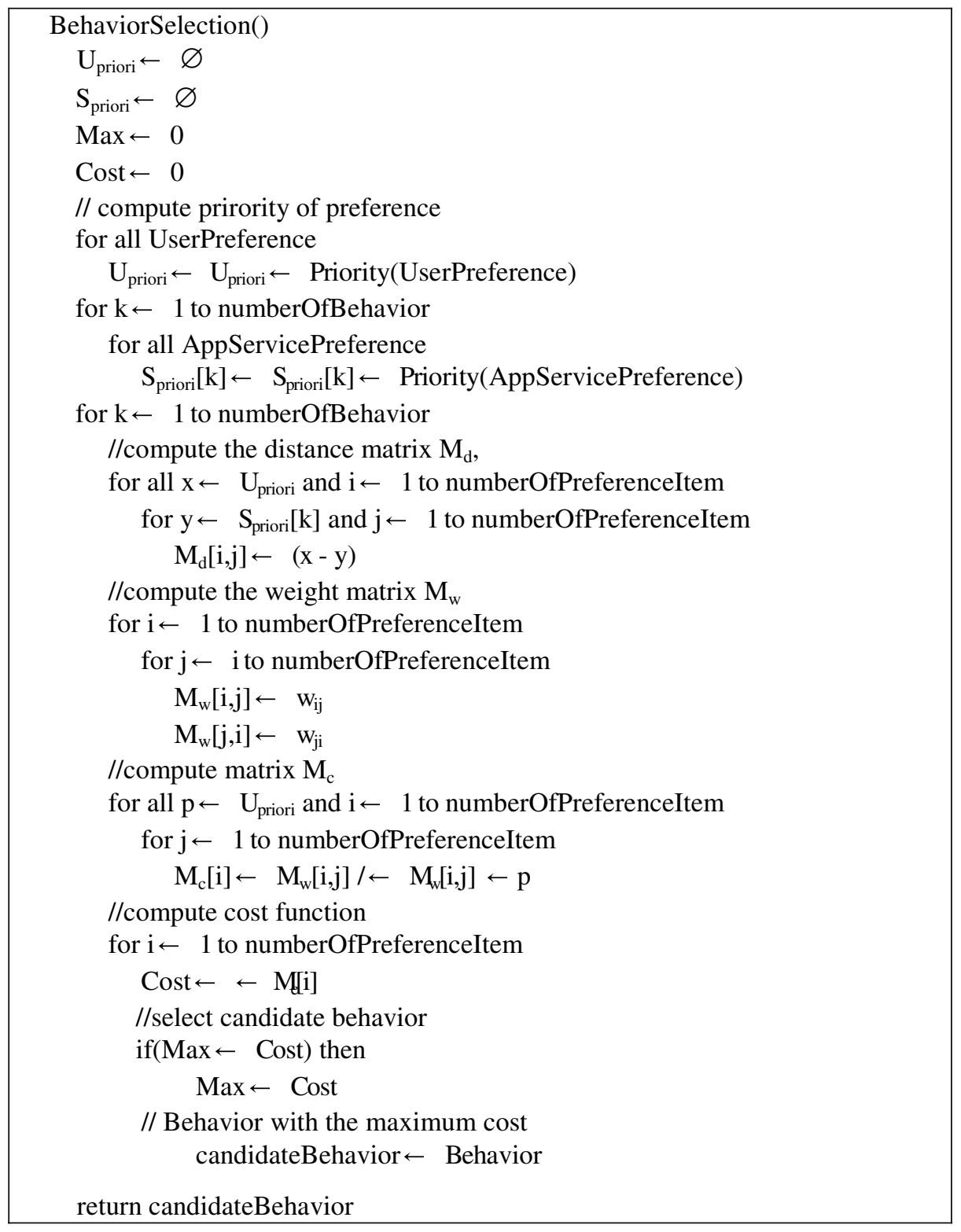

Fig. 5. High level adaptation for the behavior selection 


\subsection{Adaptation Method}

With triggered-adaptation, we support an application adaptation for context changes; context-changes trigger adaptation. The way of adaptation is carried out when an event is driven by applications. Applications can dynamically change the set of possible behaviors, as well as the associations between these behaviors and their corresponding enabling contexts, in order to cope with varying application needs and unpredicted context conditions. For the adaptation, we propose behavior decision algorithm that is composed of behavior selection algorithm and behavior mediation algorithm.

\subsubsection{Behavior Selection}

Generally mobile users would require higher QoS as possible with the minimal resource consumption, because mobile devices have the limited computing and network resources. At the selection stage, the adaptation manager in middleware measures the fitness of application to user requirements for regulating the tradeoff of these two objectives. The following describes our high-level adaptation: behavior selection (See Fig. 5). Firstly, the adaptation manager performs the ranking computation to get priority of preference, and then compute the distance matrix $\leftarrow_{\mathrm{d}}$. Each element $\leftarrow_{d}$ represents interval between user's preference and application's preference. Based on $\leftarrow_{d}$, the weight matrix $\mathrm{M}_{\mathrm{w}}$ is calculated, which represents how much $A$ is preferred than $B$. In the current work, we use experimental values for the preferred degree, $M_{w w}$ The cost matrix is $M_{w}$ computed from $M_{w}$, to valuate the fitness of application service behavior. Hence, the behavior with the maximum cost is selected as the candidate behavior.

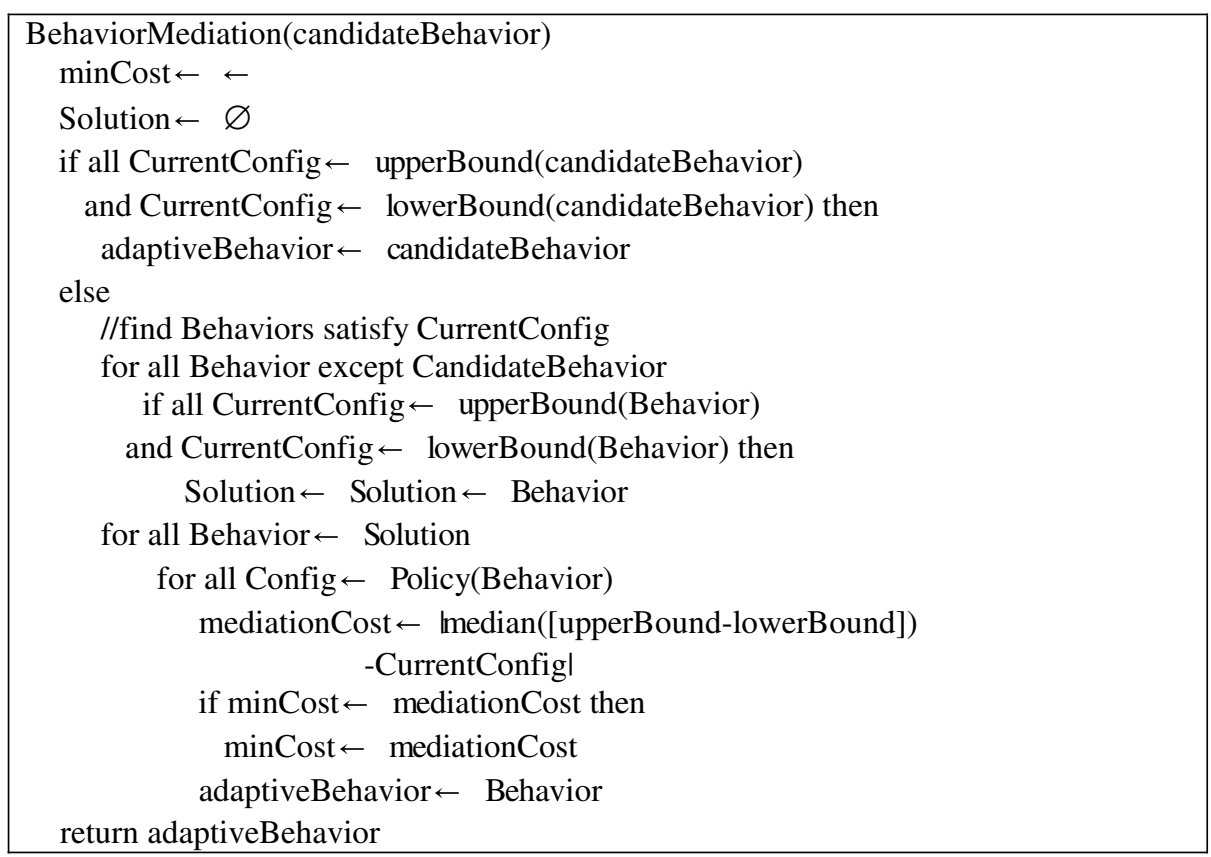

Fig. 6. Low-level adaptation for the behavior mediation 


\subsubsection{Behavior Mediation}

To execute the candidate behavior selected at the previous stage, the current configuration should support this behavior. On this mediation phase, we examine the current configuration of user's device and then decide whether the candidate behavior can be executed or not. The following describes low-level adaptation: behavior mediation (See Fig. 6).

If the candidate behavior is not suitable for current conditions, the adaptation manager should find another behavior to adapt. In the other words, if the middleware has more than one behavior whose upper threshold and lower threshold satisfying current condition, the middleware compute mediationCost to select the behavior with the minimum cost as the adaptive behavior.

\section{Implementation}

We now present our current implementation of the context-adaptive middleware. We have implemented middleware engine and metadata server in Java using jdk 1.4.2 and MySQL 4.0. At this stage, we have demonstrated audio service for PDA users.

Figure 7 represents an overview of our system architecture. Configuration Manager receives configuration information from sensor nodes whenever user configurations are changed. Adaptation Manager performs the behaviour decision process using context information; preferences and configurations. Execution Manager maintains binding information of behaviors to execute services. When an adaptive behavior is selected in the result of the behavior decision, Execution Manager binds behavior objects to provide mobile users with their application services. Communication Manager is responsible for exchanging XML messages to store or retrieve metadata.

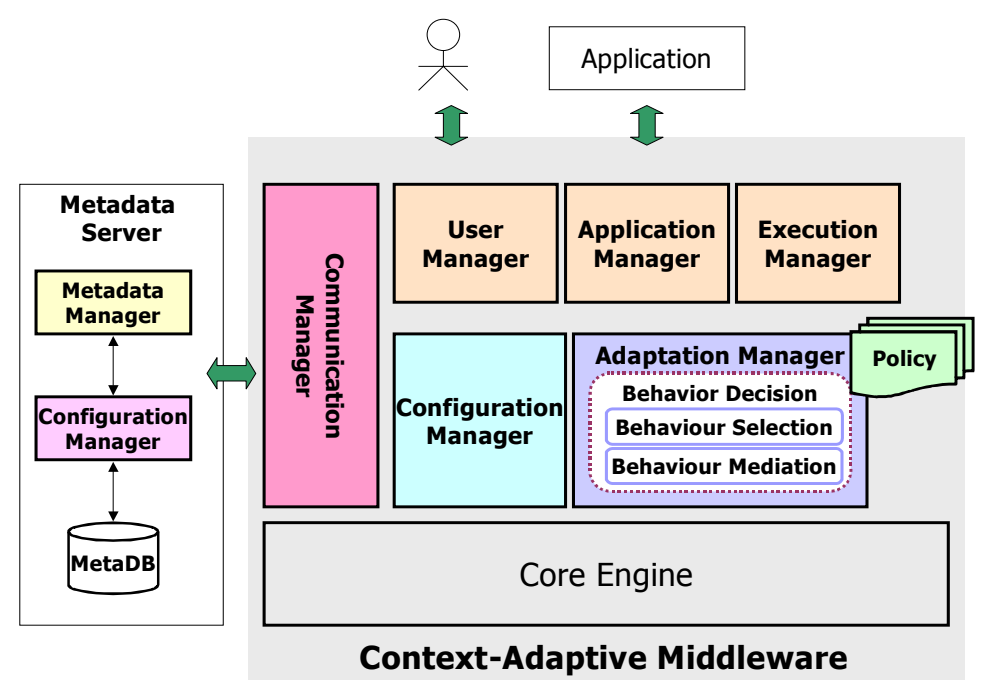

Fig. 7. Context-Adaptive Middleware Architecture 


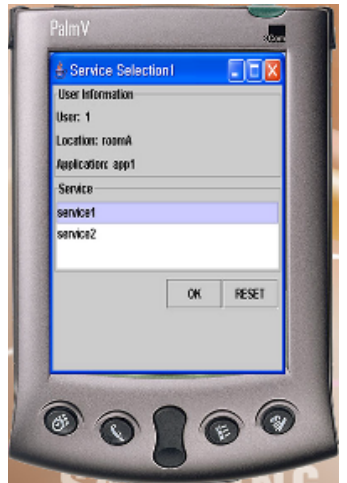

(a)

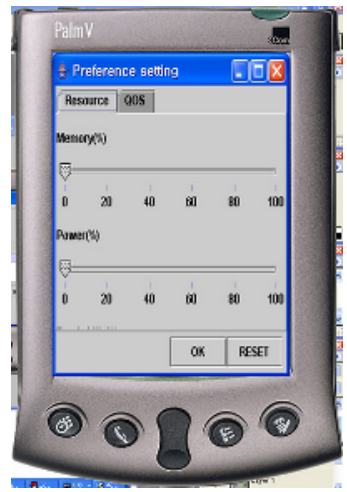

(b)

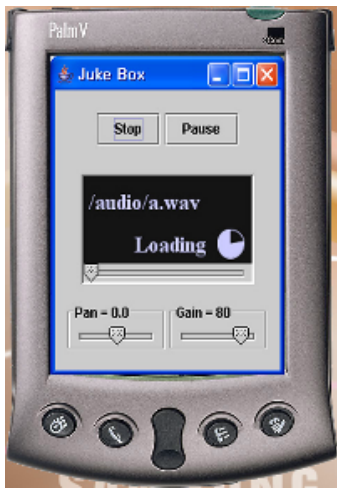

(c)

Fig. 8. View of Implementation Results. (a) Service Selection, (b) Preference Setting, (c) Service screen.

In Figure 8, there are shown three kinds of implementation results. Figure 8(a) shows the interface of service selection and Figure 8(b) shows the interface of preference setting for user requirement. The screen in Figure 8(c) shows the loading of contents to support an application service by managing of the context-awareness middleware.

\section{Conclusions and Future Work}

The context awareness is the essential technology that middleware must be equipped with in the ubiquitous environment where various computing unit are connected through a various types of network in order to provide continuous service to a user. Therefore, the middleware for applications in the ubiquitous computing environments should easily acquire context information from various context sources and should provide service adapted to context changes.

In this paper, we defined a context itself and described the design of context-adaptive model based on component for applications in ubiquitous environments. The context adaptive model can support dynamic adaptation for mobile users and applications using the context such as high-level and low-level information. We proposed a policy-based adaptation method using mobile agents in context-awareness middleware. The adaptation policies can be specified by the user-specific and application-specific priorities on a user's preference to applications and quality of services, and an application's resource requirements. The implementation of our adaptive middleware framework is currently ongoing, focusing on supporting context-aware mobile applications. In the future work, we intend to develop adaptive middleware services and management mechanism for context information. The adaptive model will be used for a contents rendering function that is a part of context-awareness middleware.

Acknowledgement. This research was supported by IRC(Internet Information Retrieval Research Center) in Hankuk Aviation University. IRC is a KyounggiProvince Regional Research Center designated by Korea Science and Engineering Foundation and Ministry of Science \& Technology. 


\section{References}

1. A. K. Dey, D. Salber, and G. D. Abowd, A conceptual framework and a toolkit for supporting the rapid prototyping of context-aware applications. Human-Computer Interaction, Vol. 16, 2001.

2. A. Ranganathan and R. H. Campbell, A Middleware for Context-Aware Agents in Ubiquitous Computing Environments, In ACM/IFIP/USENIX International Middleware Conference, Rio de Janeiro, Brazil, June 16-20, 2003.

3. G. S. Blair, G. Coulson, A. Anderson, et. al., A Principles Approach to Supporting Adaptation in Distributed Mobile Environments. Proceedings of the 5th International Symposium on Software Engineering for Parallel and Distributed Systems (PDSE'2000), Nixon P. \& Ritchie I. (eds), Limerick, Ireland, June 10-11, 2000.

4. Z. J. Haas, Designing Methodologies for Adaptive and Multimedia Networks, IEEE Communications Magazine, pp. 106-107, Vol. 39, N.11, November 2001.

5. A. Liotta, A. Yew, C. Bohoris, and G. Pavlou, Supporting Adaptation-aware Services through the Virtual Home Environment, Proceedings of the 9th Workshop of the HP OpenView University Association, June 11-13, 2002.

6. C. Efstratiou, K. Cheverst, N Davices and A. Friday, An Architecture for the Effective Support of Adaptive Context-Aware Applications, Proceedings of the Second International Conference on Mobile Data Management (MDM '2001), pp. 15-26, January 8 - 10, 2001.

7. M. Román, F. Kon, and R. H. Campbell, Reflective Middleware: From Your Desk to Your Hand, IEEE Distributed Systems Online Journal, Special Issue on Reflective Middleware, Vol. 2, No. 5, 2001.

8. B. Noble, System Support for Mobile, Adaptive Applications, IEEE Personal Communications, Vol. 7, No. 1, February 2000.

9. Capra, L., Blair, G. S., Mascolo, C., Emmerich, W., and Grace, P., Exploiting Reflection in Mobile Computing Middleware, ACM Mobile Computing and Communications Review, 6(4), pp. 34-44, 2003. 